\title{
On modelling the structural quasiness of complex systems
}

\author{
GIANFRANCO MINATI \\ Italian Systems Society \\ Via Pellegrino Rossi, 42 \\ ITALY \\ http://www.gianfrancominati.net/
}

\begin{abstract}
Complex systems are usually represented by invariant models which at most admit only parametric variations. This approach assumes invariant idealized simplifications to model these systems. This standard approach is considered omitting crucial features of phenomenological interaction mechanisms related to processes of emergence of such systems. The quasiness of the structural dynamics that generate emergence of complex systems is considered as the main feature. Generation achieved through prevalently coherent sequences and combinations of interactions. Quasiness (dynamics of loss and recovery, equivalences, inhomogeneity, multiplicity, non-regularity, and partiality) represents the incompleteness of the interaction mechanisms, incompleteness necessary even if not sufficient for the establishment of processes of emergence. The emergence is extinguished by completeness. Complex systems possess local coherences corresponding to the phenomenological complexity. While quasi-systems are not necessarily complex systems, complex systems are considered quasi-systems, being not always systems, not always the same system, and not only systems. It is addressed the problem of representing the quasiness of coherence (quasicoherence), such as the ability to recover and tolerate temporary levels of incoherence. The main results of the study focus on research approaches to model quasicoherence through the changing of rules in models of emergence. It is presented a version of standard analytical approaches compatible with quasiness of systemic emergence and related mathematical issues. The same approach is considered for networks, artificial neural networks, and it is introduced the concept of quasification for fixed models. Finally, it is considered that suitable representations of structural dynamics and its quasiness are needed to model, simulate, and adopt effective interventions on emergence of complex systems.
\end{abstract}

Key-Words: - Coherence, Emergence, Incompleteness, Interaction mechanism, Quasification, Quasiness.

Received: June 27, 2021. Revised: November 30, 2021. Accepted: December 12, 2021. Published: December 23, 2021.

\section{Introduction}

The purpose of this article is to consider approaches that model complex systems. This includes collective systems, intended as generated, emergent through designed, detected, or inferred multiple interaction mechanisms (combinations of interactions).

The approach detailed here considers quasiness, defined as generic specification, attribute the acquisition of emergent properties and processes (as interaction mechanisms). This includes inhomogeneity, multiplicity, non-regularity, partial synchronizations and partially different combinations. Quasiness is considered a feature of interaction mechanisms (idealized or inferred) and structural dynamics (the processes of change over time). Is the predominant aspect of quasiness, indeterminacy and incompleteness (given, for instance, by incomplete occurring of a process terminated early; incomplete initial conditions; incomplete iterations; partial consideration of the values of variables and their combinations) characterizing real phenomenological interactions of emergence? Determination and completeness are ideal simplifications for modelling essential aspects of phenomena. Idealized modelling, considered reliable since it captures crucial properties, fails to consider quasiness.

In this study, such quasiness is considered as predominant property in modelling phenomena and mechanisms of emergence of complex systems when the purpose is to act on them, e.g., induce, orient, vary, deactivate, and merge them. At this regard it is considered how previous research investigates the 
theoretical incompleteness of emergence phenomena [1].

Quasiness of complex systems emergent from multiple interaction mechanisms is characterized by quasi-coherence (quasiness of coherence, intended, in short, as long-range correlation and scale freeness, see Section 4.1). Understood as dynamic sequences or combinations of coherences of variable ranges, their loss and recovery, inhomogeneity and irregularity. The dynamics of local, possibly globally temporal, coherences in processes of emergence [2] is related to the countless instantaneously equivalent configurations of elements. For example, flock of birds, equivalent with regard to the different combinations of interacting entities. More precisely, quasi-coherence is a property of the nonlinear changing of rules constituting multiple interaction mechanisms generating emergence. This is in contrast to fixed or equivalent structural rules that allow stable configurations with only parametric change.

Multiple changing rules of the interaction mechanisms represent phenomenological structural dynamics that are only partially considered in fixed parametric models. Simulations focus on properties rather than on their processes of acquisition and of emergence.

The article introduces examples of mathematical approaches useful for ideal modelling of quasiness of complex structures and multiply emergent systems. In Section 2, there are definitions of some introductory concepts useful for the following topics: interaction mechanism, structural dynamics, selforganization, emergence, grey systems, fuzzy systems, multiple systems, quasiness, complex systems and theoretical incompleteness. In Section 3, there is a concise overview of approaches to modelling complex systems.

In Section 4, there is the proposal of analytical and network modelling of quasiness and introduction of the quasification of fixed models. In particular, in Section 4.1 considers consolidated approaches to modelling, representing coherence, dealing with Synchronized Multiple Synchronizations, Local Couplings, Covariance, Correlation and Crosscorrelation. Section 4.2 presents mathematical proposals for analytic and network modelling of quasiness. Subsection 4.2.1 considers classic models based on fixed rules. Subsection 4.2.2 introduces approaches to modelling based on variable rules. This proposal is an analytical approach to modelling the quasiness of the structural dynamics of phenomenological interaction mechanisms supporting emergence. Subsection 4.2.3 considers how this approach may be also implemented with networks having changing linkage. Subsection 4.2.4, considers the possibility to quasify models based on fixed rules. What is introduced in the three previous subsections is a theoretical background for modelling the quasiness of complexity. Subsection 4.2.5 presents a general view on the novelties introduced. Section 5 presents a final summary. Finally, section 6 introduces issues for further research.

Briefly, the original contributions of the article are as follows:

- When simulating a complex system, it is insufficient to generate an artificial system that possesses certain properties characteristic of that system. It is necessary to properly simulate interaction mechanisms, allowing suitable reactions to structural interventions. For example, models of complex systems showing correlation, scale-freeness, and power laws may adequately recreate features insufficient to model the interaction mechanisms supporting emergence and reactions to external interventions. In particular, it should be stressed that correlations do not imply causality [3]. It is also a matter of recognition of the inadequacy of pursuing unique, optimum, complete models of complex systems constructed of time-dependent variables instead of modelling with timedependent rules. This is related to the general quasiness of complex systems. That is, their being not always systems and not always the same systems. The model's properties change over time, even through multiple coherences are present when scale-freeness endures.

- Modelling complex systems is focused on the theoretical incompleteness of quasiness of their detected, inferred, ideally modelled, or represented phenomenological interaction mechanisms generative of the emergent characterizing properties. Not on their characteristic properties alone. Such models are here, however even if at different levels and modalities, constituted of time-dependent rule sets, for example, systems of differential equations or network linkages. These represent quasiness of structural dynamics of complex systems. Modelling in this manner is assumed to be suitable to simulate not just complex systems behaviours and their acquisitions of properties (rather than acquired properties) but also the proper reactions to external interventions in their entirety and not only in reference to specific properties.

- The variability of rules charactering models of quasiness of complexity. Section 4.2 introduces examples of related mathematical approaches, 
both analytical and based on the science of networks. There is the introduction of the concept of quasification for fixed models.

This is the fundamental conceptual innovation proposed in this article.

The conclusions stress that understanding how complexity can be generated and represented by structural dynamics in the context of the quasiness, rather than assuming invariable representations very far from the dynamics and multiplicity of reality, enables the design of more suitable and realistic models of the system.

\section{Introductory concepts}

To aid the reader, this section briefly summarizes some conceptual aspects in this research area. Namely, characteristics of complex systems and related concepts. In the literature, these concepts are widely elaborated. Here, there is the reviewing of the concepts of interaction mechanism, structural dynamics, self-organization, emergence, grey systems, fuzzy systems, multiple systems, quasiness, complex systems and theoretical incompleteness. Citations are abridged from the available literature to those useful later in the article.

\subsection{Interaction mechanism}

The elementary understanding of 'interaction' occurring between pairs, is that "one's behaviour depends on another's behaviour". On the concept of interaction, Von Bertalanffy wrote [4, p. 19]:

"Application of the analytical procedure depends on two conditions. The first is that interactions between 'parts' be nonexistent or weak enough to be neglected for certain research purposes. Only under this condition, can the parts be 'worked out', actually, logically, and mathematically, and then be 'put together.' The second condition is that the relations describing the behavior of parts be linear; only then is the condition of summativity given, i.e., an equation describing the behavior of the total is of the same form as the equations describing the behavior of the parts; partial processes can be superimposed to obtain the total process, etc. These conditions are not fulfilled in the entities called systems, i.e., consisting of parts 'in interaction.' The prototype of their description is a set of simultaneous differential equations, which are nonlinear in the general case. A system or 'organized complexity' may be circumscribed by the existence of 'strong interactions' or interactions which are 'nontrivial'non-trivial', i.e., nonlinear." (See the system of simultaneous differential equations (1) reported in section 3.2).

An interaction mechanism is given by multiple combinations of interactions. An example of generic interaction mechanism is given by the irregular combinations of single interactions in Brownian-like motions and gasses. Interaction mechanisms of interest here are those that support acquisitions of coherences and processes of emergence. Dealing with simulated flocks of boids, beside elementary assumptions and constraints (such as imposing collision avoidance, cohesion rules, alignment rules as in Reynold's modelling, see subsection 4.2.4), there is assumption of anisotropic flocking where a case of interaction mechanism is given by the occurrence of interaction rules applied by sequences of boids chosen in any way, such as the elementary: if the speed of the closer boid is greater, less or equal to $k$, then correspondingly keep, increase or reduce of a suitable parameter $w$ the speed.

Countless variations of such rules are possible. For instance, by context-sensitive computing $k$, the rule may apply only to specific boids having speed greater or less than $h$; considering the average speed of the $n$ closer boids; replacing metrical closeness, for instance, with topological closeness; replacing speed with altitude or replacing speed with direction, and their possible combinations, to be applied generally or depending on parametric values, etc.

Interaction mechanisms may be considered in a phenomenological context of applied organizations constituting structured configurations and rules. Hierarchical, horizontal, functional, matrix and reticular organizations are possible. There are plenty of examples such as the military complex, corporate entities and commercial institutions where rules may apply in different context-sensitive ways. That is, for example, commercial rules may be applied in different ways depending on the products produced and how they are marketed. Other examples include organized games such as artificial intelligence games considered by the game theory [5-7]. In these games, formalization is possible and permits simulation of properties.

Another approach to understanding interaction mechanisms is allowing for self-organizing processes. These constitute partial regular sequences of varied, contextually applied configurations of interaction rules. For instance, partially repeated and synchronized processes. Some examples include selfconstitution of patterns in Cellular Automata and the establishment of whirlpools in liquids and air (hurricanes). Again, formalization is possible and allows behavioural simulations. 
Yet another approach to interaction mechanisms considers the emergent processes as constituted by coherent sequences of partial, combined, varied, lost, and recovered configurations of interaction rules. The multiple interaction mechanisms of emergence of collective systems refer to the occurrence of interactions. For example:

- Variable over time,

- Multiple interactions between the same and different elements,

- Between multiple elements (clusters),

- In combinations,

- At different intensities,

- With different, and time-varying, start and duration times,

however sufficient to keep significant subsequent levels of coherence. The interaction mechanisms of emergence can have properties such as their regular or partial recurrence; also including their evolution and mutation, combination and ability to generate levels of coherence and multiple local coherences. Such properties may characterize specific processes of emergence, as is the case with flock-, swarm-like behaviours and biological life.

Formalization is more difficult and only possible for specific properties, for example, for artificial life. The multiplicity of interaction mechanisms of emergence may be modelled by considering dominant aspects such as correlations and selfsimilarity. The focus here is on the dominant aspect of quasiness in structural dynamics and in the occurrence of multiple interaction mechanisms. Quasiness in models, as in quasiness of correlations and self-similarity, are expected to make these mechanisms less ideal but more effective and realistic.

Furthermore, quasiness is expected to increases the understanding of collective phenomena in addition to facilitating structural modifying interventions on collective phenomena.

The three cases considered above are not precisely separated and may occur in variable combinations.

\subsection{Structural dynamics}

The introductory concept of structural dynamics is considered in different ways, including sequential structural changes of cytoskeletons [8, p. 89]. Different modes of interaction assumed by individual agents in collective behaviours, complex systems intended as cascades and sequences of phasetransition-like changes $[9,10]$. In general, structural dynamics is considered a constituent of acquisitions, changes, losses, and combinations of structures and interaction mechanisms. These occur, for instance, through phase transitions and networks properties [8, p. 87-102].

\subsection{Self-organization}

Self-organization is assumed to occur when a population of interacting entities acquires collective sequences of properties in a phase-transition-like manner. Having regularities and stabilities, such as dominant repetitiveness, and synchronicity. The occurrence of interaction mechanisms has some regularities such as partial, but predominant, iterations in a context of quasiness. Examples include the behavioural patterns of mosquitoes swarming around a fixed light and whirlpools $[8$, p. 33-37; 1114].

\subsection{Emergence}

A population of collectively interacting entities is assumed to establish processes of emergence when acquiring sequences of properties in coherent ways, they generate long-range correlations. The process of emergence may be understood as the occurrence of possibly multiple simultaneous sequences of processes of self-organization when the corresponding acquired dynamic structures are coherent (a case is given by the theory of 'dual evolution' for adaptive systems, introduced by Paperin et al. [15], see also [8]. Emergence may be understood as a generalization of self-organization in which partial regularities, synchronization, and stabilities are substituted by coherences. The multiple sequences of interaction mechanisms of emergence generating and supporting complex systems have quasiness as main feature. That is, their non-regular recurrence, inhomogeneous applications, partially occurring, evolution and mutation. Furthermore, this includes combinations and the ability to generate levels of coherence, and multiple local coherences. Examples include collective emergent systems such as anthills, cities, flocks, the internet, markets, networks, social systems and swarms. Emergent systems keep their coherences and are robust to perturbations $[2,8,16-20]$.

\subsection{Grey systems}

Grey systems are characterized by incompleteness and uncertainty in measurements, information about composing elements, structures, boundaries, interaction mechanisms, and the system's behaviours $[21,22]$. Their incompleteness is not theoretical, as it can be completed (see point 2.10). 


\subsection{Fuzzy systems}

Fuzzy variables are those whose value is specified as an allowable range rather than a single value. For example, $0<x<1$ instead of $x=0$ or $x=1$ [23-26]. Fuzziness deals with properties of values under the assumptions of stability and invariability of the structural representations of the phenomena. Not about the variability of structures and their properties, as in the case of multiple interaction mechanisms. It may be a matter of changing the fuzziness level of interacting agents. Fuzzy systems use fuzzy values and dealing with incomplete information. Examples include incomplete or partially incorrect words for search engines, and uncertain measurements.

\subsection{Multiple systems}

A multiple system is considered to be a set of systems whose components simultaneously belong to more than one system [8, p. 104]. For example, when multiple networks are constituted by the same nodes belonging to different and simultaneous networks [27]. When multiple states of belonging occur, some multiple systems can be fuzzy. Cases of multiple belonging to different systems can occur through multiple dynamical and contextual multiple contextual roles. This is the case, for instance, when the same components correspondingly play, simultaneously and at different times, unintended multiple roles and give rise to the emergence of different systems. For instance, when different components may be simultaneously part of different systems and their behavior is intended to play different independent roles. For instance, a behavior may play a functional role and at the same time be source of information, used to dissipate, and as regulatory such as in biology and electronic devices, and a price has different roles in economical systems. Another case occurs when considering interchangeabilities between elements due to their levels of equivalences (equivalent for some systems but not for other). We stress that multiple belonging and interchangeable roles are in this case passive (same properties has multiple meanings, effects) for components, while they nay may be active when components assume multiple interacting (in a flock same boid interacts by changing speed, position and direction).

\section{8 @uasiness}

The following instances are examples of the concept of quasi in the scientific literature. Quasicrystals take a particular solid form where atoms are arranged in a structure that is deterministic. They are not periodic or repetitive as can be observed in normal crystals.
There are patterns where the local arrangement of the material is regular and stable but not periodic throughout the material. The characterizing property is incompletely respected in multiple possible ways [28]. In thermodynamics, quasistatic processes occur slowly enough to allow the system to remain in internal thermodynamic equilibrium. That is, the volume changes so slowly that the pressure remains uniform. In physics, quasiparticles possess traditional particle properties with the exception of localization [29]. In mathematics, quasiperiodicity relates to recurrences whose periodicity has components that are irregular or unpredictable.

Quasiness attributes specifically to the generic dynamics of the occurrence of incompleteness in phenomena of emergence. This is particularly the case in collective phenomena where countless equivalences occur. For example, in the behavioural multiplicity of global and local patterns of spatial positions assumed by single interacting agents (such as boids in flocks), densities, distributions, acquired patterns. It is the assumption of local dynamic configurations that makes dispositions that had lost their coherence become temporary coherent again [8, 2]. As dealt with at the end of this subsection, this is the case for components of a collective behaviour acquiring ergodicity as an involuntary consequence of their movement [30]. Components of populations are intended to assume ergodic behaviours if their behaviour is such that when, at any moment in time, $x \%$ of the population is in a particular state, then each component of the population spends $x \%$ of time in such state. Ergodicity is a recurrent property of statistical systems. However, this is a formal and absolute definition of ergodicity. In real cases, there is consideration of percentages that establish significant levels of ergodicity when components assume percentages of same roles at different times, and simultaneous different roles, but with the same percentages establishing quasi-ergodicity.

In the multiple dynamics of emergent phenomena, as in collective behaviours, large varieties of instantaneous configurations of elements (e.g., boids in flocks) are equivalent. For a flock, there are countless equivalent configurations of the same flock. Basically, the quasiness specifies the separation from simplified, ideal representations used for fixed models, from the phenomenological processes. Such differences are usually neglected by idealized models as irrelevant. Here it is considered as predominant in modelling phenomena and mechanisms of emergence of complex systems. The quasiness is related in several ways and levels by which collective phenomena, may globally, partially and locally assume, lose, recover and reassume levels 
of coherences. The ability to recover and tolerate temporary levels of incoherence occurring in predominant or non-predominant properties of collective systems, where the occurring of tolerance may be measured in terms of percentages, their variations, periodicity, and other regularities. This is different from considering tolerance thresholds. This is a matter of robustness and resilience of collective behaviours.

In processes of emergence, quasiness represents the possibility of the structural dynamics of their interaction mechanisms to recover, acquire and temporarily gain inhomogeneous local coherences $[8$, p. 153-154]. The attribution of quasiness may be conceptually generalized to various properties in correspondence with their non-complete, nonregular, specific and inhomogeneous occurrences. This includes indefinite combinations of phenomenological events and processes. Ideal models focus on the essential characterizing properties of a phenomenon, such as the scale freeness of a collective behaviour. In contrast, the focus is on how these properties are achieved and maintained. The interest is not in averages only, but on properties of the distributions leading to such average. In addition to the convergence point, it should be also considered the convergence process. For instance, it is possible to consider a variable $x$ with the constraint $0<x<1$. How constraints are respected is as important as the constraint itself. That is, sequences of values of the variable $x$ close to the end points, or having regular fluctuations between them, or fluctuations around the mean. In the following, the cases of quasi-systems and quasicoherence and other concepts introduced later are mentioned.

Quasisystems (for which there is quasiness in being systems) are intended as sequences (be they coherent, temporarily incoherent, resuming the same or different coherence) of different (possibly fuzzy), inhomogeneous versions of the same system [8]. Quasisystems can involve any level of fuzzy belonging, and be locally, or temporarily, not systems nor the same systems. Furthermore, quasiness can occur when the same system may operate in both fuzzy and non-fuzzy ways. Fuzziness in a quasisystem can take the form of indeterminate numerical parameters describing its state in the class of systems. In contrast, the quasiness of systems is structurally specified by not always being systems, not always the same system, and not only systems, by their incomplete processes of resumption and recovering of properties. As is the case for coherence, multiple structural dynamics and multiple interaction mechanisms in collective systems. Fuzziness, on the other hand, deals with indeterminacy in numerical indices of state. Quasiness is concerned with the specification of processes (here interaction mechanisms supporting emergence). A system is not 'quasi' when its multiplicity is reduced to one or very few systems. However, in some special cases a quasisystem may be approximated by a single or few systems. Quasisystems are very realistic and require suitable approaches, considering and not neglecting their quasiness as it is used in simplified modelling. Subsection 4.2.2 presents possible approaches.

Quasicoherence (having quasiness in coherence) is characterized by dynamic coherences of local or variable range, coherence loss and recovery processes, combinations of coherences, and coherences as with long-range correlations. The latter can be represented by a graphical example of a stylized flock as quasicoherent quasisystem having quasiness of multiple complex behaviours, (see Fig. 1). Modelling phenomena of quasicoherence may include those of remote synchronization based on indirect information transfer. This occurs when nonadjacent pairs of entities become substantially synchronized despite there being no direct structural connections between them [31-33]. At this regard it is possible to consider the case when the coherence of an emergent collective behaviour is given (locally, partially, temporarily) by the occurrence of ergodicity (see, for instance, [8, p. 161-170; 34, 35]. The same system, or parts thereof, can be both ergodic and non-ergodic depending upon the time scale of the observer. For example, polymers or even temporarily ergodic systems. As in the concept of quasi-ergodicity as related to degrees or indices of ergodicity $[8$, p. 118,162$]$ recognized as another example of quasicoherence.

\subsection{Complex systems}

Complex systems are systems generated by, and in which, multiple processes of emergence occur. Complex systems are therefore quasisystems having predominant, multiple coherences. In contrast to noncomplex systems, they do not acquire the same systemic property over time. They implement continuous processes that establish coherent structural changes in acquiring properties and behaviours. Multiple irregularities of quasiness can be recognized as belonging to multiple processes of temporary loss, recovery, or partial coherences. That is, acquisition of properties that adapt and restore forms of coherence to values that had become incoherent. Examples of acquired properties are belonging to the basin of an attractor, correlation (long range), network properties, polarization and global ordering, power laws, remote 
synchronizations, scale invariance, and selfsimilarity. Examples of complex systems include climate systems, dissipative structures, double pendulums, flocks, swarms and social systems. They have multiple properties that vary in value such as long-range correlations and scale invariance [36-39].

\subsection{Theoretical incompleteness}

Incompleteness occurs as a phenomenologically and consequently theoretically necessary condition of the models, even if it is not sufficient for the assumption of coherences and multiple emergences, in the emergence of complex systems.

Emergence complex systems are intended as theoretically incomplete since a single model is not sufficient for their representation; the system variables (degrees of freedom) are variable in number and continuously acquired; non-equivalent properties are continuously acquired. The emergence of complex systems requires such theoretical incompleteness.

The generic concept of theoretical incompleteness [1, 40-42] can suitably specify the one of quasiness. Theoretical incompleteness is a property of phenomena that is incomplete enough to permit emergence of complex systems. Their multiple structural dynamics and dynamical coherences are in the context of equivalences. Completeness is an 'enemy' of emergence as it produces single emergence without leaving a role to equivalences [43].

Theoretical incompleteness, as theoretical noncompletability, can be ascribed, for instance, to:

- The Uncertainty Principle in quantum mechanics, by which accuracy in measuring one variable is at the expense of another,

- Complementarity in theoretical physics, for example, between wave and particle natures,

- Partial acquisitions, loses, and recovery of properties in processes of emergence in dynamics of equivalences,

- The incompleteness theorems introduced by Gödel in 1962,

- Partial or non-decidability,

- Non-computable uncertainty when considering that probabilities must relate to variable configurations of events and not to improbable isolated abstract events [44, 45],

- Non-complete, non-explicit, non-univocal and non-equivalent modelling. Such as the DYnamic uSAge of Models (DYSAM) based on the Bayesian method, statistical approaches of 'continuous exploration' of events and ensemble learning as in [8, p. 201-204; 46, p. 64-75].

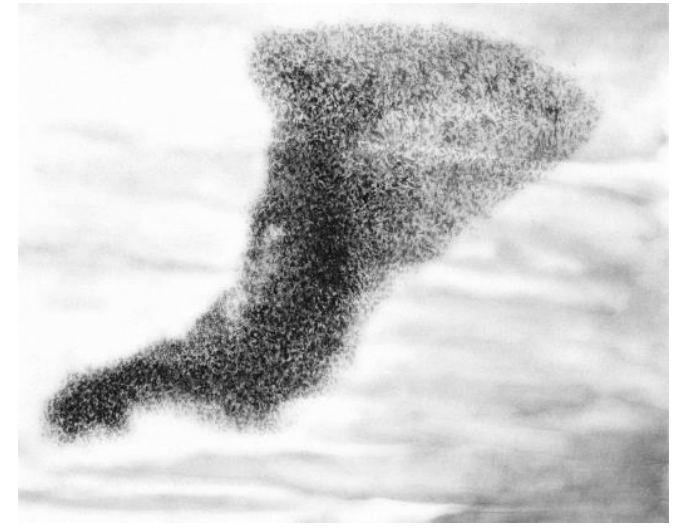

Fig. 1 Graphical example of a stylized flock having quasiness of multiple complex behaviours.

\section{Modelling complex systems}

This section presents a concise overview of approaches to model complex systems. There is mention of the following approaches:

a) Considering ideal models that generate data and assume properties have significant similarities with the real phenomena [47-50]. This is especially true for simulations. For instance, properties of nondeterministic chaos are studied mainly through simulations.

b) Considering real data related to spatial positions of entities interacting in 3D. For instance, when interacting entities are equipped with a global positioning system (GPS). As used in cars, when tracking animals in herds, economic values (for instance prices and share values). This includes their macroscopic properties such as density and acquired properties such as scale invariance. There is the consideration of ideal models that work with real data and are interpolated to model behaviours [51-54]. The models here are considered simply as 'models of the system'.

c) Ideal models are considered consisting of inferred constraints by the possible interaction mechanisms and derived rules of interaction [55-60]. The models are considered to represent the real generative interaction mechanisms of the system and be suitable for simulations.

d) The high level of dispersion, (global) single low interpolation and the suitability of multiple interpolations of real data, indicate phenomenological intense structural dynamics. In addition to multiple processes of emergence. This requires the use of sequences of nonequivalent, different ideal (characterized by a top-down structure and based on general 
principles assumed to be universally valid) and non-ideal (for instance, data-driven -statistical-, properties of interpolations, based on artificial learning and combinations of general principles -optimization- and specific choices requiring computer simulations) models. This is the research issue dealt with in this article. The ideal and non-ideal models are considered as having significant correspondence with the real generative interaction mechanisms of the system and share their essential, possibly necessary but not always sufficient, features of quasiness.

The focus is on multiple modelling and little on increasing and optimizing the levels of approximation and behavioural simulation (cases a and $b$ ). The interaction mechanisms and their crucial feature of theoretical incompleteness are represented by quasiness. This allows for simulations of realistic reactions to structural changes, external influences and adoption of adequate approaches to act on the phenomenon (cases $\mathrm{c}$ and $\mathrm{d}$ ). Currently, this is conducted by acting on parameters of fixed models (cases a and b). They are considered reductionist and inappropriate to represent the real phenomenon and its interaction mechanisms.

In this article, the focus is on cases c) and d) as approaches to be considered when actions are to be implemented on complex systems. Such as economic, environmental, medical, and social systems and where appropriate modelling is necessary.

\section{Approaches to model coherence, structural dynamics and quasiness. Quasification}

Section 4.1 considers consolidated approaches to model and represent coherence in the cases of Synchronized Multiple Synchronizations, Local Couplings, Covariance, Correlation, and CrossCorrelation. On the other hand, Section 4.2, presents mathematical proposals for analytic and network modelling of quasiness. Such proposals are analytical approaches developed to model the multiplicity and quasiness of multiple interaction mechanisms, and their variable but predominant coherences in processes of emergence.

\subsection{System data value coherence}

Global, local, and multiple coherences are detectable. For instance, in scale-free correlations among quantitative and measured properties of system components. This is without considering or needing to know how the interaction mechanisms are modelled or their structural dynamics.

The detection of coherences is very important. They are significant clues to the existence of consistent interaction mechanisms of emergence. Which, in turn, are important to hypothesize about and implement interventions on the phenomenon. Usually, the approach is to use a single model that is either assumed to correspond to a single fixed idealized interaction mechanism or sufficient to approximate. This averages the effects of more than one interaction mechanisms. Such an approximation is considered as a matter of reductionism, since it neglects structural dynamics of the interaction mechanisms. Such reductionism may be acceptable in the simplest cases but not generalizable to highly complex systems. For these systems the level of complexity is given, for instance, by the number and dynamics of the processes of emergence occurring, the acquired properties, and the quasiness.

Approaches to detect coherence are neither descriptive nor prescriptive of the interaction modalities of the components. In our view, the occurrence of multiple quasicoherences is to be understood not in terms of liability or approximations that may possibly be refined, but as corresponding to multiple composed and superimposed phenomenological interaction mechanisms.

As considered in Section 3.3, the research challenge is to realize suitable approaches to model generative interaction mechanisms and their dominant properties such as quasiness. This should fit phenomenological data rather than trying artificially generate data usually achieved through simulations that approximate effects and properties of the phenomena (cases a and b, in Section 2).

Let us now turn to well-represented cases of coherence [61]. In the conceptual framework of theoretical incompleteness, quasicoherence may be understood to be represented as the irregular occurrence of dominant coherences and in their combination. Related research approaches concentrate on detection of such quasicoherence and infer crucial characteristics of the corresponding possible interaction mechanisms. These approaches focus on the interaction mechanisms and their properties instead of confusing simulation of their effects. This confusion occurs between the generating mechanisms and their effects. For example, by considering the geometrician of cobwebs made by spiders intended as pursued on purpose having some geometrical-like abilities instead of behavioural effects. This is also the case for bees that build space-occupation-optimized hexagonal cells in their hives, or the birds that build 
certain kinds of nests. Even animals that establish particular kinds of colonies. The effort to artificially recreate the outcomes of this behaviour ignores the interaction mechanisms generating such behaviour.

\subsubsection{Synchronized multiple synchronizations}

The phenomenon by which various kinds of synchronizations are established, which in turn become synchronized, has been observed in the human nervous system and for populations of chaotic systems [11, 38, 62-64]. If the higher-level synchronization of multiple local instantaneous synchronizations is maintained, then it can be considered as a form of coherence [62]. An example of a model of these phenomena is given by considering ensembles of globally coupled chaotic maps (see, for instance [65, p. 155]. The coherence of their dynamics is described by laws of the form

$$
x_{i}(n+1)=(1-\varepsilon) f\left(x_{i}(n)\right)+\frac{\varepsilon}{N} \sum_{j=1}^{N} f\left(x_{j}(n)\right)
$$

where:

- $\quad N$ is the number of chaotic maps,

- $\quad i=1, \ldots, N$ is a space index,

- $\quad x_{i}(n)$ denotes the value of the $i$ th map in correspondence to the discrete time $n=$ $0,1, \ldots$,

- the function $f(x)$ is given by $f(x)=$ $a x(1-x)$ (logistic map) where:

- $\quad a$ denotes the non-linearity parameter of the logistic map and

- $\varepsilon$ denotes the coupling parameter.

\subsubsection{Local couplings}

It is possible to consider more complicated systems in which the couplings are local rather than global. This is the case for chains of coupled limit-cycle oscillators, see [66], described by equations having a generic form of the kind

$$
\dot{\varphi}_{n}=\omega_{n}+F\left(\varphi_{n}\right)+d\left(\sin \left(\varphi_{n+1}-\varphi_{n}\right)+\sin \left(\varphi_{n-1}-\varphi_{n}\right)\right.
$$

where:

- $\varphi_{n}$ denotes the phase of the $n$th oscillator,

- $\omega_{n}$ its natural frequency,

- $d$ a suitable parameter and

- $F\left(\varphi_{n}\right)$ denotes a nonlinear function responsible for the non-uniformity of rotations of the considered oscillator.

\subsubsection{Bravais-Pearson coefficient}

It is generally possible to use measures of correlation by applying the linear approach of the so-called Bravais-Pearson coefficient [67], see [68] for a review. This quantity measures the linear correlation between two sets of data. Namely, the covariance of two variables being divided by the product of their standard deviations. Considering that covariance determines the extent to which two random variables, denoted by $X$ and $Y$, covary. That is, the way they change in the same way [69]. The Bravais-Pearson coefficient is essentially its normalized measurement (with values between -1 and 1 ). This coefficient, as covariance itself, measures only linear correlations and neglects other types of relationships [70-72].

Given a pair of random variables $(X, Y)$, in a population, Pearson's correlation coefficient $\rho$ is given by

$$
\rho(X, Y)=\frac{\operatorname{Cov}(X, Y)}{\sigma X \sigma Y}
$$

where:

- Cov is the covariance,

- $\sigma X$ is the standard deviation of $X$ and

- $\sigma Y$ is the standard deviation of $Y$.

Covariance is given by

$$
\operatorname{Cov}(X, Y)=\frac{\sum(x-\bar{x})(y-\bar{y})}{n}
$$

where:

$$
\begin{aligned}
& -\bar{x} \text { and } \bar{y} \text { are the means of the data series } \\
& \text { and } \\
& -n \text { is the size of the considered sample. }
\end{aligned}
$$




\subsubsection{Cross-Correlation function}

The Bravais-Pearson approach is generalized by other linear measures, including the cross-correlation function. Let us consider two time series of length $N$ whose normalized values are denoted by $x_{n}$ and $y_{n}$ with zero mean and unitary variance. The crosscorrelation function $C_{X Y}(\tau)$ depends on time lag $\tau$ and varies within the range from $-(N-1)$ to $N^{-1}$ according to the following law:

$$
\begin{aligned}
& C_{X Y}(\tau)=\frac{1}{N-\tau} \sum_{n=1}^{N-\tau} x_{n+\tau} y_{n} \quad \text { if } \quad \tau \geq 0 \\
& C_{X Y}(-\tau) \quad \text { if } \quad \tau<0
\end{aligned}
$$

Cross-correlation values can run from 1 (maximal synchronization) to -1 (anti-phase signals). In conceptual agreement with the issues discussed above, it is possible to consider quasicoherences as partially occurring in the cases mentioned above. They are varying locally and valid over time in all possible combinations.

\subsection{Analytical and network modelling of quasiness. Quasification.}

This section considers two classic mathematical approaches considered suitable to model structural dynamics and quasiness. This includes systems of differential equations and networks. The problem is to consider idealized approaches to model multiple, different, inhomogeneous, local or long-range, continuous or temporary changes of the interaction mechanisms occurring in emergent complex systems.

\subsubsection{Fixed rules}

In classical approaches to complex systems, components are assumed to be in fixed numbers and to interact continuously in the same nonlinear way. The exception being parametric variation. Moreover, acquired systemic properties are assumed to be generated by fixed models. The classical approach utilizes techniques to interpolate, model by considering data properties, preform conceptual simulations and employ idealistic fitting models. This approach almost inevitably ignores the real processes and their properties. It is considered to be approximated by ideal assumptions and abstractions validity of abstract long-range unexplained correlation, chaotic behaviours, power laws and polarization [43]. The real generative interaction mechanisms and their properties, such as quasiness, are rarely inferred and most often remain unknown and neglected. They are assumed to be suitably represented by fixed models and the properties of models from data [2].

Conversely, in this study, the focus is on considering (see subsection 4.2.2) representations and properties of changes in the compositions of the applied interaction mechanisms. Such representations and properties are indispensable and predominant characteristics of phenomenological interaction processes leading to constituent emergence. This approach is supposed to allow realistic simulations in the presence of environmental changes and external perturbations. This also enables more of a systemic structural understanding that is needed to design interventions on complex systems.

An example of the first case (fixed rules) is given by the classic analytical definition of a system, denoted by $S$, as mentioned in paragraph 2.1. Consisting of $n$ interacting elements $p_{i}$ for which there exist some measurements $Q_{i}(i=1,2, \ldots, n)$. In the simplest case, such as the occurrence of a finite and stable number of elements, $S$ may be ideally identified by instantaneous $Q_{n}$ values and by their time evolution. This is represented by a system of coupled, firstorder, ordinary differential equations $[4$, p. 56],

$$
\left\{\begin{array}{l}
d Q_{1} / d t=f_{1}\left(Q_{1}, Q_{2}, \ldots Q_{n}\right) \\
d Q_{2} / d t=f_{2}\left(Q_{1}, Q_{2}, \ldots Q_{n}\right) \\
\ldots \ldots \ldots \ldots \ldots \ldots \ldots \ldots \ldots \ldots \\
d Q_{n} / d t=f_{n}\left(Q_{1}, Q_{2}, \ldots Q_{n}\right)
\end{array}\right.
$$

The assumption is that model (1) identically and generally applies to modelling systems. The interaction mechanism is also considered coinciding with system (1). It is supposed to model the phenomenological structural dynamics in a simplified and reduced way and, therefore, its effects on properties of $p_{i}$ are directly related to $Q_{n}$.

Among the many possible examples, it is possible to mention the well-known Lotka-Volterra equations [73]. This models a system of interacting prey and predators

$$
\left\{\begin{array}{l}
d x / d t=a x-c x y \\
d y / d t=-b x+c x y
\end{array}\right.
$$

where $x$ is the density of prey individuals, $y$ the density of predators, $\alpha$ is the intrinsic rate of preypopulation increase, $\beta$ denotes the predation rate 
coefficient, $\gamma$ the predator mortality rate and $\delta$ the reproduction rate of predators per eaten prey.

Another well-known example is given by the socalled Lorenz equations [74] that model the occurrence of deterministic chaos. That is, sensitive dependence on initial conditions is the essence of deterministic chaotic systems:

$$
\left\{\begin{array}{l}
\frac{d}{d t} x(t)=\sigma(y-x) \\
\frac{d}{d t} y(t)=r x-y-x z \\
\frac{d}{d t} z(t)=x y-b z
\end{array}\right.
$$

where $r, b$, and $\sigma$ are control parameters.

The above is included in the conceptual framework adopted by various models of collective behaviours. For example, flocks, swarms, fish schooling simulators, see [47, 50, 51, 53, 75-80].

Our approach is to focus and identify highrepresentative macroscopic laws that are assumed appropriate to approximate the phenomenological interaction mechanisms leading to microscopic behaviours of $p_{i}$.

Often, such high-representative laws (correlations, power laws, scale invariance and self-similarity and statistical distributions) are assumed first as models of the generative interaction mechanisms. They are then considered as the structural dynamics of the phenomena. This may be acceptable for a certain type of simulations where the approximation is sufficient for studying. This is the case for evolutionary phenomena, and special cases such as strange attractors, distribution and convergence. On the other hand, it is misleading when used to act by using such laws, understood as models of the generative interaction mechanisms. This can lead to confusion over the acquired properties, processes of acquisition of properties, and properties of the interaction mechanisms.

\subsubsection{Variable rules}

At this point it is possible to consider approaches representing structural variations, for instance when in (eqs.6) $f_{n}$ changes in $f_{n, t}$ as in the system of equations (eqs.9),

$$
\left\{\begin{array}{l}
d Q_{1} / d t=f_{1, t}\left(Q_{1}, Q_{2}, \ldots Q_{n}\right) \\
d Q_{2} / d t=f_{2, t}\left(Q_{1}, Q_{2}, \ldots Q_{n}\right) \\
\ldots \ldots \ldots \ldots \ldots \ldots \ldots \ldots \ldots \\
d Q_{n} / d t=f_{n, t}\left(Q_{1}, Q_{2}, \ldots Q_{n}\right)
\end{array}\right.
$$

(eqs. 9)

The meaning of time in $f_{n, t}$ (eqs.9) lies in the fact that the functio(i) $f_{n}$ has the possibility to change in different instants but, however, keeping the same role, in this case to specify the value of the variable $d Q_{n} / d t$.

The crucial aspect is that the temporal systemic interdependence between $f_{n, t}$, the mutual definition in terms of each other of $d Q_{n} / d t$, preserves their coherence. In an ideal system (eqs.9), the structural significance of time is related to the selection of valid $f_{n, t}$, or of the transient non-applicability of any of them, or the usage of new nonequivalent but admissible ones. This, for example, is the case in nonsmooth transitions. Consequently, it is possible to determine in time $t$ the variables $Q_{n}$. This includes the related variables of $f_{n, t}$ and the possible transient noninvolvement in $f_{n, t}$ of some $Q_{k}(0 \leq k \leq n)$. It is possible to consider $Q_{k}=0$ until the complete noninvolvement when $k=n$ leads to the situation as represented in the system of equations (eqs.10) when momentary new variables $Q_{n+1}$ and $f_{n+1, t}$ are considered. Such dynamics in representations is considered to be corresponding to processes of structural dynamics.

However, due to the dynamics, variability and fuzziness of the phenomenological multiple interaction mechanisms there is the occurrence of a situation which is analytically intractable. The variability, the sequences of $f_{n, t}$ analytically represent the quasiness of the multiple interaction mechanisms. In modelling the quasiness of phenomenological dynamics of quasicoherent collective phenomena, specific variable sets of dominant interaction rules are considered. In this way, quasiness is modelled by considering such sets of rules. It is possible to select mechanisms depending on their ability to represent the real phenomenon.

Realistically, by implementing simulations with available data there should be consideration of context-sensitive processes from combinations of previously used $f_{n, t .}$. For example, this can be achieved using optimization criteria, and artificial learning processes, through Recurrent Neural Networks (RNN) [81]. It is possible to figure out the possibility of identify generic approaches with effective simulations. This can then be used to anticipate characteristic behavioural aspects of 
specific categories of complex systems. Furthermore, in the inferring or selection of $f_{n, t}$ occurring on real data, it is possible to consider cases in which sequences of data available are locally interpolated.

By using appropriate approaches, it is possible to consider local sequences with significant interpolation and infer or select suitable local and partial $f_{n, t}$. It is one thing to interpolate the global evolutionary path of the system (for example of the variables $Q_{n}$ ) but it is quite another to identify the $f_{n, t}$ that model partial sequences of the system. They should correspond to the occurrences of different interaction mechanisms. Where there are multiple and partial interpolations, their coherence is phenomenological. The challenge is to suitably represent this situation. This is the case when local polynomial interpolation fits many polynomials within specified overlapping neighbourhoods. These solutions may be optimized by using suitable algorithms.

This finally leads to a possible formalization of structural variability, as in the ideal system of equations (eqs.10),

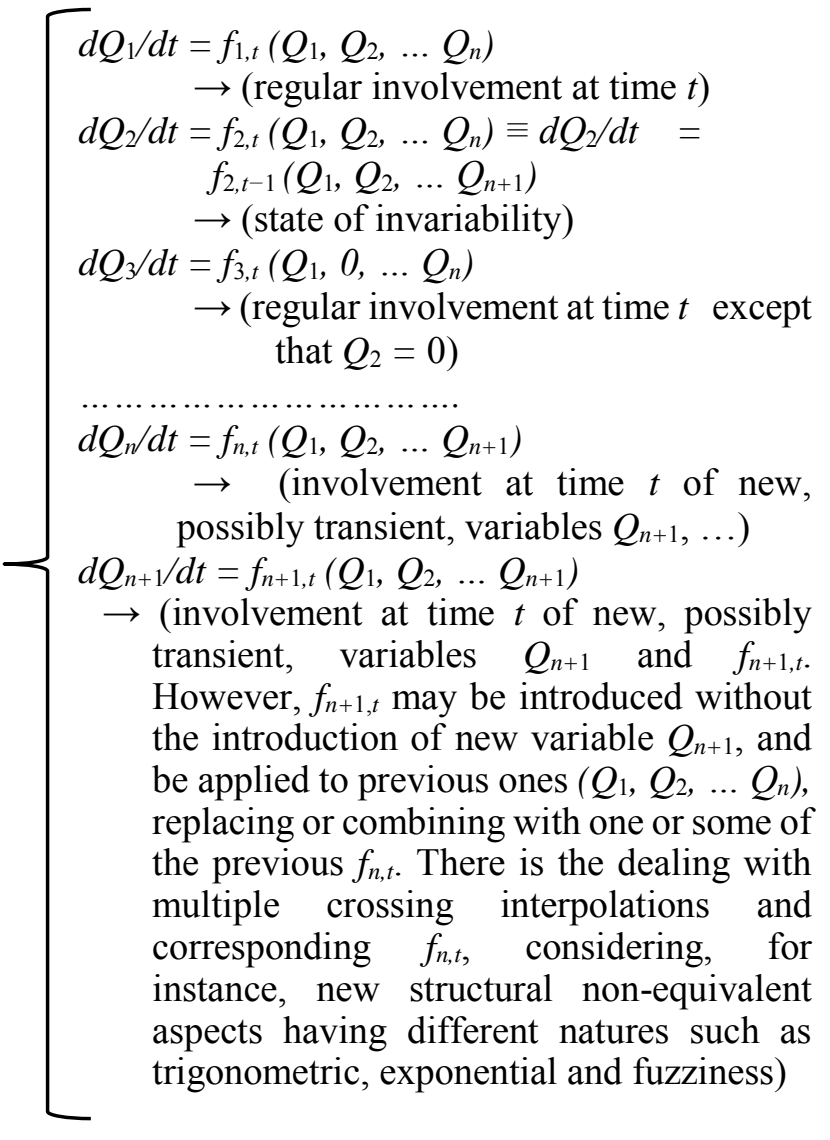

The eqs. 10 specify the characteristics of the modelling system proposed as approach. The possible changing of the variables considered is related to the fact that complex systems have many, variable degrees of freedom and variable interaction mechanisms.

Therefore, the same complex system may be modelled by different versions of the systems of ordinary differential equation of type (eqs.10). In this case, there are multiple and simultaneous, possibly overlapping, models that when taken together constitute effective modelling over time.

Modelling emergence of highly complex systems using (eqs.10) correspond to the multiplicity of the generating interaction mechanisms having quasiness as main feature. That is, their non-regular recurrence, inhomogeneous applications, partial occurring and possible combinations. Coherence is given by the constraint of being in a system of equations. Moreover, the variety of models of the type (eqs.10) may not apply to the whole system homogeneously. This depends on the real phenomenology and areas of the system which are modelled according to variants of the model. Some examples of zones include the boundary and central parts of the system, as in the dynamics of flocks or swarms.

\subsubsection{Networks}

The analytical representations depicted by (eqs.10) have an interesting conceptual correspondence to network representations of complex systems. The Science of Networks [82-85] represents systems as networks and systemic properties as network properties. The quasi and irregular roles of the equations in (eqs.10) corresponds to situations when the linkages between nodes over time are not static. That is, irregular, context-sensitive, non-linear and weighted. This situation may occur in a variety of ways, for instance, when networks are scale-free. That is, having a high number of nodes with few links or a small number of nodes with a high number of links. If the small-world property holds, then most distant nodes can be reached from every other node via a small number of intermediate links. This situation depicts the occurrence of quasi-networks: networks having variable cluster coefficients, degree distributions and fitness. The quasiness of networks is introduced in [86].

Other approaches may consider combinations of analytical and non-analytic computational processes, such as artificial neural networks (ANN). This is also the case for networks changing levels and number of nodes, such as RNN, (see Fig. 2). This is achieved by using internal states to process sequences of inputs, nature-inspired computational approaches [87], and changing class in cellular automata. The reference is to the classic four classes considered by Wolfram [88] for the evolution of Cellular Automata characterized by: 1) evolution towards a spatially 
homogeneous equilibrium state; 2) evolution toward stable or periodic attractors with finite spatial extent; 3 ) the possibility of chaotic evolutions with unlimited spatial growth of initial patterns; and 4) occurrence of localized patterns having great complexity with the ability to grow and contract.

Systems of the type (eqs.10) and variable linkages in Networks are considered to represent the dynamics of quasiness in general and, in particular, multiple interaction mechanisms.

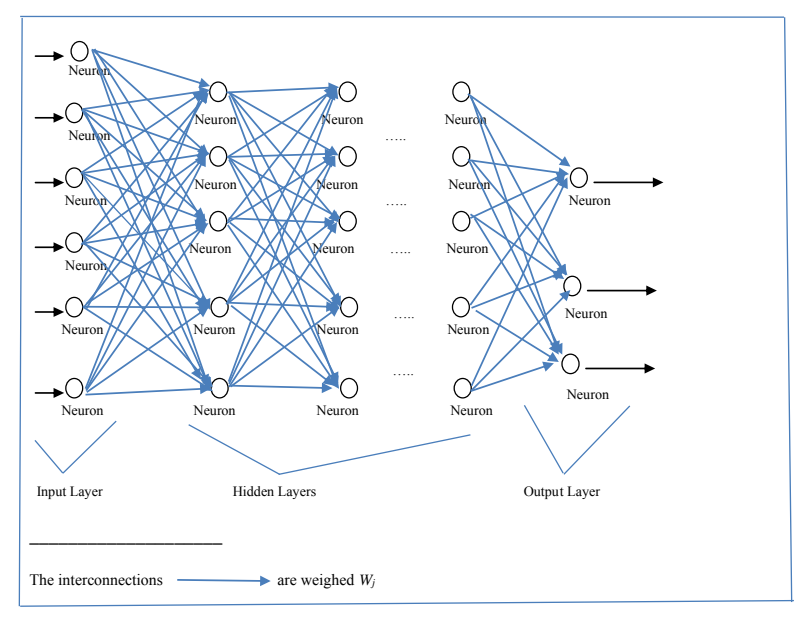

Fig. 2 A schema of an artificial neural network with changing layers

\subsubsection{Quasification}

At this point, it is possible to consider the process of quasification for the usual fixed models and constraints. Conceptually it is a matter to replace fixed properties with their inhomogeneity, multiplicity, non-regularity, and partiality of different combinations.

Quasification may consist of analytical and network models transformed into non-continuous sequences and variable combinations of interaction rules and linkages. The quasi nature of the system is specified by the properties of such sequences and combinations. This includes, partial recurrences (with non or partial periodicity), random recurrences and inhomogeneous occurring (interaction rules and linkages relate to variable significant percentages of the system's components).

Regarding varying constraints, it is possible to consider the behavioural constraints assumed by the well-known Reynold's model [54]. The interaction mechanism being an ideal modelling of flock generations. In Reynold's model, the constraints of the interacting mechanisms for individual components (birds) imply that they must:

- Have motion pointing towards the average direction of locally adjacent components (alignment rules),

- Avoid the crowding of locally adjacent components (separation rules) and

- Point towards the average position of locally adjacent components (cohesion rules).

There are many varieties of modalities by which such constraints may be respected (allowing quasiness) that further facilitate coherence and emergence. However, this configuration of constraints (having whatever formalizations) may be further quasified when such binding rules apply in different ways (generally or differently by areas or subsets of interacting entity). For example, with partial regularities, in variable combinations and with different parameters. It should be noted that quasification does not lead to increasing generality or indefiniteness. It does, however, lead to the specification of quasiness of properties of specific collective behaviours.

Similarly, it is possible to consider the occurrence of quasi-deterministic chaotic systems and quasi LotkaVolterra systems applied in a specific way. In the same way, it is possible to quasify systems of inferred rules such as interactive behavioural models of pedestrians, crowd formation, vehicle traffic and shoaling fish. Of course, it is possible to consider the reverse processes of 'undoing' quasification by reducing the considered sequences of options until they adopt a single fixed configuration. This is in conceptual alignment with the processes that transform a set into a fuzzy set (and vice versa).

\subsubsection{A general view}

Multiple interpolations and systems of ordinary differential equations of type (eqs.10) conceptually correspond to the usage of multiple modelling of phenomena of emergence. This is established by multiple interaction mechanisms that are noncompletely analytically representable modellable. Their modelling requires simultaneous multiple nonequivalent models as considered by the DYnamic uSAge of Models (DYSAM). For example, it is possible to mention simulations requiring multiple usage of different ANN, considered in [46, p. 76-85]. Here these models are realistic, either DYSAM-like or not (when the number of ANN used is just one). For the latter, the modelling is quasi-DYSAM.

In collective behaviours they are not properly reducible, but primarily not understandable when considered being due to parametrical changes in the fixed model of equations used as a general model of 
collective behaviours (cases a and $b$ in Section 3). While partial simulations may be implemented with such reductions, i.e., the use of single fixed generic models, simulations of experimental interventions require more proper modelling that considers not only approximate structural dynamics.

The cases mentioned for system (eqs.10), of Section 4.2.2, are considered to be examples of models of structural changes in the interaction mechanisms. In collective behaviours, they are irreducible and primarily not understandable as due to parametrical changes in the fixed model of equations used as a general model of collective behaviours (cases a and $\mathrm{b}$, in Section 3). While partial simulations may be implemented with such reductions, using single fixed generic models, simulations of experimental interventions require proper modelling beyond approximate structural dynamics.

A proper modelling should be used when considering flocks or swarms under attack from a predator and under sudden environmental perturbations. A tentative example amenable to this approach is available on-line [89], see also [90]. This is related to a flock simulator designed on the basis of a specific generative interaction mechanism. In this case, the well-known Reynold's model, (see Section 4.2.4), for implementing simulations where at each instant all microscopic spatial information related to each single boid is available.

Multiple systems establishing coherent collective behaviours represent cases modelled as superimposed quasi-simultaneous systems. As in the cases of multiple systems of ordinary differential equations (eqs.10), in Section 4.2.2, and multiple linkages in networks, in Section 4.2.3.

In models of collective behaviours there is the need to deal with the establishment of coherence and quasicoherences represented by the occurrence of various phenomena. This includes scale-free correlations among measures of various properties that occur at similar times. Moreover, for significant percentages of components such as directional, metrical, topological, and mediated information transfer [91, 48].

The conceptual symbolic case consisting of multiple simultaneous and possibly crossing systems of ordinary differential equations (eqs.10) and networks may have a variety of different versions. This includes the use of sub-symbolic [9] ANN (the networked, weighted, occurring at different levels, computational processing of ANN is considered nonexplicit and non-analytically represented. For this reason, it is called sub-symbolic, whereas the ANN program is an explicit algorithm, see Fig. 2), natureinspired [87, 92, 93] computational approaches and their combinational change over time. This is expected when multiplicity relates to the effective generative interaction mechanisms. The multiple interaction mechanisms, such as given by $f_{n, t}$ in systems (eqs.9) and (eqs.10), (in Section 4.2.2), and their quasiness are considered conceptually equivalent to the inferred generative interaction mechanisms (see Table 1 for a concise view).

1) Ideal fixed models acquire characterizing properties of complex systems. For example, attractors, behavioural, coherences, correlation and power laws.

2) Ideal fixed models on multiply interpolated real data and constraints.

3) Ideal fixed models of inferred interaction mechanisms, constraints suitable to simulate crucial features and properties of complex systems.

4) Ideal and quasified fixed models acquiring characterizing properties in quasi ways. That is, changes in number, type and applicability of equations. This occurs in multiple, simultaneous, possibly overlapping systems of ordinary differential equation (9) and in a sequence of partial networks. Changing the rules, their domain of application and succeeding each other is considered to correspond to the structural dynamics and quasiness of the phenomenological generative interaction mechanisms. An example is the quasification of simulation models, e.g., agentbased models.

5) Ideal and quasified fixed models on interpolations of real data; designed, detected, inferred constraints. It is a matter of ideal models of the generative interaction mechanisms where the focus is on the designed, detected, or inferred multiple interaction mechanisms and constraints.

6) Non-ideal models as a mixture of general principles and of specific choices. For instance, data-driven and based on artificial learning, properties of interpolations, Big Data approaches and constraints to the interaction mechanisms detected from real data of different nature. Examples are found in 3D data collected using GPS, stereo-metric and computer-vision applications and economic datasets. Typical examples are profiles, behavioural standards, models as formalisations of phenomenological constraints to the interaction mechanisms (inferred rules of interaction for vehicular traffic) and shoaling fish. Such non-ideal models are characterized by quasiness representing their structural, phenomenological, and (irregularly) coherent dynamics.

Table 1 Ideal fixed models; ideal quasified fixed models; non-ideal models. 


\section{Final summary}

Standard research and simulation approaches aim to identify the better fitting interpolative model. Generating the acquired properties that are supposed to approximate to the best the phenomenological real mechanism. Under certain conditions, the phenomena of quasiness and quasicoherence, if applicable, are assumed to be negligible as microscopically irrelevant on the macroscopic bestfitting model. These conditions apply when dealing with populations of generative interaction mechanisms that are intractable by their inhomogeneity, limited period of validity, irregularities and non-repeatability in the application. This situation corresponds to the microscopic intractability of components when suitable strategies are designed to look for statistical properties. (Such as molecules of a gas, for which statistical thermodynamics considers only systems of very large numbers of molecules and neglects details of individual behaviours).

It is assumed that such omissions are admissible when considering microscopic data values for components and their acquired properties. This approach, nevertheless, hides the structural dynamics of fundamental importance to the design and execution of interventions to modify an emergent complex system hence, permitting the interpretation of structural interventions on the interaction mechanisms. While reductionist assumptions, such as the admissibility of separability, completeness and finiteness may be contextually effective, they cannot be generalized, thus, making emergent properties and mechanisms of emergence theoretically invisible [8, p. 359].

In the absence of hypotheses about, or knowledge of, the structural dynamics phenomena modifying interventions are reduced to non-structural, symptomatic, interventions on properties of the components and on the parameters of the interpolative models. It is also a matter of reducing complex systems to non-complex systems and assuming a suitable simplified approach, which is often self-defeating [94]. This coincides with the inability to represent and manage complex phenomena such as those that occur in economic, environment (e.g., climate and territorial safety), medical (e.g., pandemics and migratory capacity and resistance in collective aggregations of tumour cells leading to metastases), and social (e.g., criminality) settings.

Other approaches consist of collecting data from real phenomena such as using stereo-metric digital photogrammetry data related to real flocks [48], data provided by GPS systems, data from ad hoc electronic devices of coupled oscillators generating emergence $[95,96]$, and data of different natures, such as collective phenomena in economics and of signals. Such data are usually interpolated, and their collective properties are assumed to be validating properties of the interpolative models.

Real, phenomenological interactive mechanisms, such as for living beings $[97,98]$ are not intrinsically, formally, representable and analytically intractable if not simplified and idealized. However, the nonexplicit phenomenological interaction mechanisms may be represented by some of their supposed dominant critical features. In this regard, the article considered approaches such as systems of differential equations as in (eqs.10) and quasi-networks that can represent the quasiness of structural dynamics, supposed as critical systemic feature of phenomenological interactive mechanisms supporting emergence of collective systems. This approach may be applied in reverse to quasify phenomenological interactions.

The property of quasiness has been considered as represented by properties of systems of inferred analytical models of critical feature, as item of an ideal list to be extended by future research since phenomenological coherence remains a matter of research, because: "Whatever the origin of the scalefree behaviour is, ..., the fact that the correlation is almost not decaying with the distance, is by far the most surprising and exotic feature of bird flocks. How starlings achieve such a strong correlation remains a mystery to us. " [48] (Cavagna et al., 2010).

\section{Further research}

Research should focus on developing approaches for multiple modelling using multiple crossed systems of type (eqs.10) integrated with other sub-symbolic approaches. This includes ANN with variable, hidden layers, number of neurons, networks with variable linkage and nature-inspired computations. There was the introduction of conceptual approaches and lines of research to be implemented by corresponding models and simulations for experimentation.

It may be fruitful to consider approaches based on identifying explicit and equivalent possible functions $f_{n, t}$, based on machine-learning techniques, game theory, optimization techniques, analogue processing, and clustering techniques allowing to consider clusters [8, p. 102-116] rather than microscopic entities.

The challenge is to develop suitable approaches to model generative interaction mechanisms that fit the quasiness of phenomenological data. Such methods facilitate appropriate structural interventions on 
complex systems. The focus should be on ideal or inferred models of effective generative interaction mechanisms and their quasiness. However, it might possibly also proceed by identifying categories of phenomenological interaction mechanisms of complex systems. Furthermore, as has happens in physics, a long-term research perspective might be to reconceptualize systems as fields rather than as interacting entities $[8$, p. 231-233]. The problems considered here would then be completely redefined.

\section{Conclusions}

Highly complex systems in which multiple processes of emergence occur, acquiring coherent properties over time, should not be modelled by adopting the same approaches used for other systems that possess stable temporal properties. Consequently, in modelling complex systems, it is not sufficient just modelling some crucial properties. Rather, the focus should be on their acquisition processes and their features. Giving up, the search for the unique and optimal model constituted of fixed and iterated rules such as equations and structures of networks.

Dealing with complex systems, modelling and simulation of specific properties does not correspond to the simulation of the underlying structural dynamics of the system and its quasiness. The quasiness, the opportune incompleteness -allowing compatibility with processes of emergence of the models, is intended to constitute the crucial feature of multiple phenomenological interaction mechanisms. They are applied in an irregular and inhomogeneous way in the dynamics of loss, recovery and acquisition of properties. This occurs in variable ways, for which a complex system is not always a system, the same system and not just a system.

This study introduced mathematical approaches to represent such theoretical incompleteness. The quasiness of ideal and non-ideal models for detected, inferred or represented phenomenological interaction mechanisms generative of emergence of complex systems.

Importantly, as elaborated in this study, neglecting the quasiness of complex systems leads to the adoption of fixed, simplified, optimized ideal models suitable for non-complex systems or, at most, for specific properties. This omission involves taking approaches that are, at the very least, inadequate to act on the emergence of complex systems. This includes:

- Changing, regulating and maintaining acquired properties of emergent phenomena,

- Recognizing phenomena as emergent,
- Inducing phenomena of emergence in populations that are collectively interacting,

- Merging emergent phenomena,

- Inhibiting or accelerating the establishment of processes of emergence,

- Managing the compatibility between processes of emergence, and

- Varying the levels and type of quasiness.

These concerns relate to the ability to represent and manage complex phenomena that occur in examples of climatic, economic, medical and social settings. There was the presentation of some possible approaches for appropriate modelling with attention to structural dynamics.

The present research article is dedicated to the memory of Professor Eliano Pessa with whom these issues were under study and to celebrate his valuable interdisciplinary contribution and expertise in the science of complexity.

\section{References:}

[1] Minati, G. Knowledge to Manage the Knowledge Society: The Concept of Theoretical Incompleteness. Systems 2016. 4(3), doi:10.3390/systems4030026.

[2] Minati, G. Non-classical Systemics of quasicoherence: From formal properties to representations of generative mechanisms. A conceptual introduction to a paradigm-shift. Systems 2019. 7(51), doi:10.3390/systems 7040051 .

[3] Calude, C.S., Longo, G. The deluge of spurious correlations in big data. Found. Sci. 2016. 22: p. 595-612.

[4] Von Bertalanffy, L. General System Theory. Development, Applications, George Braziller: New York, NY, USA, 1968.

[5] Maynard-Smith, J., Evolution and the Theory of Games, Cambridge University Press: Cambridge, UK, 1982.

[6] Vincent, T.L., Evolutionary Game Theory, Natural Selection, and Darwinian Dynamics, Cambridge University Press: Cambridge, UK, 2012.

[7] Weibull, J.W. Evolutionary Game Theory, MIT Press: Cambridge, MA, USA, 1995.

[8] Minati, G., Pessa, E., From Collective Beings to Quasi-Systems, Springer: New York, NY, USA, 2018.

[9] Licata, I., Minati, G. Emergence, Computation and the Freedom Degree Loss Information Principle in Complex Systems. Foundations of Science 2016. 21(3), 1-19. 
[10] Minati, G., Licata, I. Emergence as Mesoscopic Coherence. Systems 2013. 1(4), doi:10.3390/systems 1040050.

[11] Boccaletti, S., Kurths, J., Osipov, G., Valladares, D.L., Zhouc, C.S. The synchronization of chaotic systems. Phys. Rep. 2002. 366: p. 1-98.

[12] Nagaev, R.F., Dynamics of Synchronising Systems, Springer: Berlin, Germany, 2002.

[13] Nicolis, G., Prigogine, I., Self-Organization in Nonequilibrium Systems: From Dissipative Structures to Order through Fluctuations, Wiley: New York, NY, USA, 1977.

[14] Pessa, E. Self-Organization and Emergence in Neural Networks. Electron. J. Theor. Phys. 2009. 6: p. 269-306.

[15] Paperin, G., Green, D. G., Sadedin, S. Dualphase evolution in complex adaptive systems. Interface 2011. 8: p. 609-629, doi:10.1098/rsif.2010.0719.

[16] Anderson, P. W., Stein, D. L. Broken Symmetry, Emergent Properties, Dissipative Structures, Life. Are they related? In SelfOrganizing Systems: The Emergence of Order, Yates, F.E., Ed., Plenum: New York, NY, USA, 1985, pp. 445-457.

[17] Cruchtfield, J.P. The calculi of emergence: Computation, dynamics and induction. Phys. D 1994. 75: p. 11-54.

[18] Johnson, S., Emergence: The Connected Lives of Ants, Brains, Cities and Software, Touchstone: New York, NY, USA, 2002.

[19] De Wolf, T., Holvoet, T. Emergence Versus Self Organisation: Different Concepts but Promising when Combined. In Engineering Self-Organising Systems: Methodologies and Applications, Brueckner, S.A., Di Marzo Serugendo, G., Karageorgos, A., Eds., Springer: New York, NY, USA, 2005, pp. 115.

[20] Scott, A., Nonlinear Science: Emergence and Dynamics of Coherent Structures, Oxford University Press: Oxford, UK, 2003.

[21] Javanmardi, E., Liu, S., Xie, N. Exploring the Philosophical Paradigm of Grey Systems Theory as a Postmodern Theory. Found. Sci. 2020. 25: p. 905-925, doi:10.1007/s10699019-09640-5.

[22] Liu, S., Yang, Y. A brief introduction to grey systems theory. Grey Syst. Theory Appl. 2012. 2: p. 89-104, doi:10.1108/20439371211260081.

[23] Klir, G.J., Yuan, B., Fuzzy Sets and Fuzzy Logic: Theory and Applications, Prentice Hall: Englewood Cliffs, NJ, USA, 1995.
[24] Tettamanzi, A., Tomassini, M., Soft Computing: Integrating Evolutionary, Neural, and Fuzzy Systems, Springer: Berlin, Germany, 2010.

[25] Zadeh, L. A. Fuzzy logic, neural networks, and soft computing. Commun. ACM 1994. 37: p. 77-84, doi:10.1145/175247.175255.

[26] Zadeh, L.A., Klir, G.J., Yuan, B. (Eds.) Fuzzy Sets, Fuzzy Logic, and Fuzzy Systems: Selected Papers by Lotfi A. Zadeh, World Scientific: Singapore, 1996.

[27] Nicosia, V., Bianconi, G., Latora, V., Barthelemy, M. Growing multiplex networks. Phys. Rev. Lett. 2013. 111: p. 058701, doi:10.1103/physrevlett.111.058701.

[28] Janot, C., Quasicrystals: A Primer, Oxford University Press: Oxford, UK, 2012.

[29] Pessa, E., The concept of particle in quantum field theory. In Vision of Oneness, Licata, I., Sakaji, A., Eds., Aracne: Rome, Italy, 2011, pp. 13-40.

[30] Minati, G. A Note on the Reality of Incomputable Real Numbers and Its Systemic Significance. Systems 2021. 9(44), doi: 10.3390/systems 9020044

[31] Gambuzza, L.V., Cardillo, A., Fiasconaro, A., Fortuna, L., Gómez-Gardenes, J., Frasca, M. Analysis of remote synchronization in complex networks. Chaos Interdiscip. J. Nonlinear Sci. 2013. 23: p. 043103, doi:10.1063/1.4824312.

[32] Nicosia, V., Valencia, M., Chavez, M., DiazGuilera, A., Latora, V. Remote synchronization reveals network symmetries and functional modules. Phys. Rev. Lett. 2013, 110, 174102, doi:10.1103/physrevlett.110.174102.

[33] Minati, L. Remote synchronization of amplitudes across an experimental ring of non-linear oscillators. Chaos Interdiscip. J. Nonlinear Sci. 2015. 25: p. 123107, doi:10.1063/1.4936791.

[34] Coudène, Y. Ergodic Theory and Dynamical Systems, Springer-Verlag: London, 2016.

[35] Minati, G. Emergence and Ergodicity: A Line of Research. In Emergence in Complex, Cognitive, Social and Biological Systems, G. Minati and E. Pessa, E. (Eds.), Springer: New York: Springer, 2002, pp. 85-102.

[36] Haken, H., Information and Self-Organization. A Macroscopic Approach to Complex Systems, Springer: Berlin, Germany, 1988.

[37] Hooker, C. (Ed.), Philosophy of Complex Systems, Elsevier: Oxford, UK, 2011. 
[38] Manrubia, S. C., Mikhailov, A. S., Emergence of Dynamical Order: Synchronization Phenomena in Complex Systems, World Scientific: Singapore, 2004.

[39] Yang-Yu Liua, Y.-Y., Slotine, J.-J., Baraba'si, A. L. Observability of complex systems. Proc. Natl. Acad Sci. USA 2013. 110: p. 2460 2465, doi:10.1073/pnas.1215508110.

[40] Liu, B. Uncertainty Theory, Springer-Verlag: Berlin, 2014.

[41] Longo, G. Interfaces of Incompleteness. In Systemics of Incompleteness and QuasiSystems, Minati, G., Abram, M.R., Pessa, E., Eds., Springer: New York, NY, USA, 2019, pp. 3-55.

[42] Longo, G. Reflections on concrete incompleteness. Philos. Math. 2011. 19: p. 255-280.

[43] Minati, G. Phenomenological structural dynamics of emergence: An overview of how emergence emerges. In The Systemic Turn in Human and Natural Sciences. A Rock in the Pond, Urbani, U.L., Ed., Springer: New York, NY, USA, 2019, pp. 1-39.

[44] De Finetti, B., Theory of Probability-A Critical Introductory Treatment, John Wiley and Sons: London, 1975.

[45] Galavotti, M.C. (Ed.), Bruno de Finetti Radical Probabilist, College Publications: London, 2008.

[46] Minati, G., Pessa, E., Collective Beings, Springer: New York, NY, USA, 2006.

[47] Bajec, I. L., Zimic, N., Mraz, M. Simulating flocks on the wing: The fuzzy approach. $\mathrm{J}$ Theor. Biol. 2005. 233: p. 199-220 doi:10.1016/j.jtbi.2004.10.003, doi:10.1016/j.jtbi.2004.10.003.

[48] Cavagna, A., Cimarelli, A., Giardina, I., Parisi, G., Santagati, R., Stefanini, F., Viale, M. Scale-free correlations in starling flocks. Proc. Natl. Acad. Sci. USA 2010. 107, 11865-11870, doi:10.1073/pnas.1005766107.

[49] Hoekstra, A. G., Kroc, J., Sloot, P. M. A. Simulating Complex Systems by Cellular Automata, Springer-Verlag: Berlin Heidelberg, 2010.

[50] Kunz, H., Hemelrijk, C. K. Artificial fish schools: Collective effects of school size, body size, and body form. Artif. Life 2003. 9: p. 237-253, doi:10.1162/106454603322392451.

[51] Aoki, I. A simulation study on the schooling mechanism in fish. Bull. Japan. Soc. Sci. Fish 1982. 48: p. 1081-1088, doi:10.2331/suisan.48.1081.
[52] Cucker, G., Dong, J.-G. Avoiding collisions in flocks. IEEE Trans. Autom. Control. 2010. 55: p. 1238-1243.

[53] Huth, A., Wissel, C., The simulation of the movement of fish schools. J. Theor. Biol. 1992. 156: p. 365-385, doi:10.1016/s00225193(05)80681-2.

[54] Reynolds, C.W. Flocks, herds and schools: A distributed behavioral model. In Proceedings of the 14th Annual Conference on Computer Graphics and Interactive Techniques, SIGGRAPH '87, Anaheim, California, USA, July 27-31, 1987, ACM: New York, NY, USA, 1987, pp. 25-34.

[55] Herbert-Read, J. E., Perna, A., Mann, R. P., Schaerf, T. M., Sumpter, D. J. T., Ward, A. J. $\mathrm{W}$. Inferring the rules of interaction of shoaling fish. Proc. Natl. Acad. Sci. USA 2011. 108, 18726-18731, doi:10.1073/pnas.1109355108.

[56] Jadbabaie, A., Lin, J., Morse, A. S. Coordination of groups of mobile autonomous agents using nearest neighbor rules. IEEE Trans. Autom. Control. 2003. 48: p. 988-1001, doi:10.1109/tac.2003.812781.

[57] Joo, J., Kim, N., Wysk, R.A., Rothrock, L., Son, Y.-J., Oh, Y.-G., Lee, S. Agent-based simulation of affordance-based human behaviors in emergency evacuation. Simul. Model. Pract. Theory 2013. 32: p. 99-115, doi:10.1016/j.simpat.2012.12.007.

[58] Katz, Y., Tunstrøm, K., Ioannou, C.C., Huepe, C., Couzin, I.D. Inferring the structure and dynamics of interactions in schooling fish. Proc. Natl. Acad. Sci. USA 2011. 108: p. 18720-18725, doi:10.1073/pnas.1107583108.

[59] Nagatani, T. (2012). Four species CA model for facing pedestrian traffic at rush hour. Applied Mathematical Modelling, 36(2): p. $702-711$.

[60] Xi, H., Son, Y.-J., Two-level modeling framework for pedestrian route choice and walking behaviors. Simul. Model. Pract. Theory 2012. 22: p. 28-46, doi:10.1016/j.simpat.2011.11.002.

[61] Schroeder, M., Fractals, Chaos, Power Laws: Minutes from an Infinite Paradise, Dover Publications Inc.: New York, NY, USA, 2009.

[62] Boccaletti, S., The Synchronized Dynamics of Complex Systems, Elsevier: Oxford, UK, 2008.

[63] Ciszak, M., Euzzor, S., Geltrude, A., Arecchi, F. T., Meucci, R. Noise and coupling induced synchronization in a network of chaotic 
neurons. Commun. Nonlinear Sci. Numer.

Simul. 2013. 18: p. 938-945, doi:10.1016/j.cnsns.2012.08.038.

[64] Kaneko, K. Clustering, coding, switching, hierarchical ordering, and control in a network of chaotic elements. Physica D Nonlinear Phenom. 1990. 41: p. 137-172, doi:10.1016/0167-2789(90)90119-a.

[65] Mikhailov, A.S., Calenbuhr, V., From Cells to Societies. Models of Complex Coherent Actions, Springer: Berlin, 2002.

[66] Osipov, G. V., Kurths, J. Regular and chaotic phase synchronization of coupled circle maps. Phys. Rev. E 2001. 65: p. 016216016213, doi:10.1103/physreve.65.016216.

[67] Drouetm, D., Kotz, S., Correlation and Dependence, Imperial College Press: London, 2001.

[68] Kreuz, T. Measures of neuronal signal synchrony. Scholarpedia 2011. 6: p. 11922, doi:10.4249/scholarpedia.11922.

[69] Pourahmadi, M., High-Dimensional Covariance Estimation, Wiley: Hoboken, NJ, USA, 2013.

[70] Dauwels, J., Vialatte, F., Musha, T., Cichocki, A. A comparative study of synchrony measures for the early diagnosis of Alzheimer's disease based on EEG. NeuroImage 2010. 49: p. 668-693.

[71] Kantz, H., Schreiber, T., Nonlinear Time Series Analysis, Cambridge University Press: Cambridge, UK, 1997.

[72] Pereda, E., Quiroga, R. Q., Bhattacharya, J. Nonlinear multivariate analysis of neurophysiological signals. Prog. Neurobiol. 2005. 77: p. 1-37, doi:10.1016/j.pneurobio.2005.10.003.

[73] Lotka, A. J. Undamped Oscillations derived from the law of mass action. J. Am. Chem. Soc. 1920. 42: p. 1595-1599, doi:10.1021/ja01453a010.

[74] Lorenz, E. Deterministic Non Period Flow. J. Atmos. Sci. 1963, 20: p. 130-141.

[75] Baglietto, G., Albano, E. V. Computer simulations of the collective displacement of self-propelled agents. Comput. Phys. Commun. 2009. 180: p. 527-531, doi:10.1016/j.cpc.2008.12.026.

[76] Helbing, D., Farkas, I., Vicsek, T. Simulating dynamical features of escape panic. Nature 2000. 407: p. 487-490, doi:10.1038/35035023.

[77] Quera, V., Beltran, F. S., Dolado, R. Flocking behaviour: Agent-based simulation and hierarchical leadership. J. Artif. Soc. Soc. Simul. 2010, 13(8), doi:10.18564/jasss.1609.

[78] Olfati-Saber, R. Flocking for multi-agent dynamic systems: Algorithms and theory. IEEE Trans. Autom. Control. 2006. 51: p. 401-420, doi:10.1109/tac.2005.864190.

[79] Takagi, T., Moritomi, Y., Iwata, J., Nakamine, J., Sannomiya, N. Mathematical model of fish schooling behaviour in a set-net. ICES J. Mar. Sci. 2004. 61: p. 1214-1223, doi:10.1016/j.icesjms.2004.07.009.

[80] Vicsek, T., Zafeiris, A. Collective motion. Phys. Rep. 2012. 517: p. 71-140, doi:10.1016/j.physrep.2012.03.004.

[81] Bianchi, F.M., Maiorino, E., Kamp_meyer, M.C., Rizzi, A., and Jenssen, R., Recurrent Neural Networks for Short-Term Load Forecasting: An Overview and Comparative Analysis. Springer: New York, NY, USA, 2017.

[82] Barabási, A.L., Linked: The New Science of Networks, Perseus Publishing: Cambridge, MA, USA, 2002.

[83] Estrada, E., The Structure of Complex Networks: Theory and Applications, Oxford University Press: Oxford, UK, 2016.

[84] Lewis, T.G., Network Science: Theory and Applications, Wiley: Hoboken, NJ, USA, 2009.

[85] Newman, M., Barabasi, A.-L., Watts, D.J., The Structure and Dynamics of Networks, Princeton University Press: Princeton, NJ, USA, 2006.

[86] Minati, G. Meta-rules for Networks. Journal on Systemics, Cybernetics and Informatics (JSCI) 2020. 18(6): p. 37-40, http://www.iiisci.org/journal/sci/FullText.asp ?var=\&id=IP097LL20

[87] Yang, X.-S., Cui, Z., Xiao, R., Gandomi, A.H., Karamanoglu, M., Eds. Swarm Intelligence and Bio-Inspired Computation, Elsevier: London, UK, 2013.

[88] Wolfram, S., A new kind of science. Wolfram Media Inc.: Champaign (IL), 2002.

[89] Available online: https://sourceforge.net/projects/msp3dfbsimul ator/?source $=$ directory $($ accessed on 15 July 2021).

[90] Minati, G. Tentative guidelines for the implementation of meta-structural and network software models of collective behaviours. 2017. pp. 16-22. Available online: http://arxiv.org/abs/1603.07174 (accessed on 9 December 2021). 
[91] Ballerini, M., Cabibbo, N., Candelier, R., Cavagna, A., Cisbani, E., Giardina, I., Lecomte, V., Orlandi, A., Parisi, G., Procaccini, A., et al. Interaction ruling animal collective behavior depends on topological rather than metric distance: Evidence from a field study. Proc. Natl. Acad. Sci. USA 2008. 105: p. 1232-1237, doi:10.1073/pnas.0711437105.

[92] Brabazon, A., O’Neill, M., McGarraghy, S. Natural Computing Algorithms, Springer: New York, NY, USA, 2015.

[93] Mac Lennan, B.J. Natural computation and non-Turing models of computation. Theor. Comput. Sci. 2004. 317: p. 115-145, doi:10.1016/s0304-3975(03)00635-2.

[94] Minati, G., The non-systemic usages of systems as reductionism. Quasi-systems and QuasiSystemics, Systems 2018, 6(3), http://www.mdpi.com/2079-8954/6/3/28

[95] Hong, H., Park, H., Choi, M. Y. Collective synchronization in spatially extended systems of coupled oscillators with random frequencies. Phys. Rev. E 2005. 72: p. 036217, doi:10.1103/physreve.72.036217.

[96] Minati, L., Experimental dynamical characterization of five autonomous chaotic oscillators with tunable series resistance. Chaos 2014 24(4): p. 043108-043114.

[97] Krause, J., Ruxton, G.D. Living in Groups, Oxford University Press: New York, NY, USA, 2002.

[98] Lesley, J., Morrell, R.J. Mechanisms for aggregation in animals: Rule success depends on ecological variables. Behav. Ecol. 2008.

19: p. 193-201.

\section{Funding: This research received no} external funding.

Competing Interests: The author declares no conflicts of interest.

\section{Creative Commons Attribution}

\section{License 4.0 (Attribution 4.0 \\ International , CC BY 4.0)}

This article is published under the terms of the Creative Commons Attribution License 4.0 https://creativecommons.org/licenses/by/4.0/deed.en _US 